\title{
Measurement Methods of Electron Emission Over a Full Range of Sample Charging
}

\author{
R. Hoffmann and JR. Dennison
}

\begin{abstract}
The electron emission properties of a material subject to incident radiation flux are key parameters in determining to what equilibrium charge a spacecraft will established under given environmental conditions. However, there is a complex relation between these emission properties and the charge built up in spacecraft insulators. Complex modeling codes have been developed to predict the potential a spacecraft will adopt as a consequence of its interaction with the space plasma. These require correct models of the electron yields as a function of charge to accurately predict the both the charge build up and the equilibrium potential of spacecraft components.

This paper focuses on different methods appropriate to determine the fundamental electronic material property of total electron yield as the materials accumulates charge. Three methods for determining the uncharged total yield are presented: (i) The DC Continuous Beam Method is a relatively easy and accurate method appropriate for conductors and semiconductors with maximum total electron yield $\sigma_{\max }<2$ and resistivity $\rho<10^{6} \Omega$-cm.

(ii) The Pulse-Yield Method seeks to minimize the effects of charging and is applicable to materials with $\sigma_{\max }<4$ and $\rho$ up to $>10^{24} \Omega-c m$.

(iii) The Yield Decay Method is a very difficult and time consuming that uses a combination of measurement and modeling to measure the most difficult materials with $\sigma_{\max }>4$ and $\rho$ up to $>10^{24} \Omega-c m$.

Data for high purity polycrystalline Au, Kapton $\mathrm{HN}$, and polycrystalline aluminum oxide ceramic are presented. These data demonstrate the relative strengths and weaknesses of each method, but more importantly show that the methods described herein are capable of reliably measuring the total electron yield of almost any spacecraft material.
\end{abstract}

\section{INTRODUCTION}

$\mathrm{T}$ The central theme of spacecraft charging is how spacecraft interact with the plasma environment to cause charging. Spacecraft materials accumulate negative or positive charge and adopt potentials in response to interactions with the plasma environment. A material's electron emission and electron yield, $\sigma$, (defined as the ratio of electron flux out of a material to the electron flux into it), determines how quickly

This study was partially funded by the NASA Solar Probe Mission through the Johns Hopkins University Applied Physics Laboratory and by the NASA Space Environments and Effects program.

Ryan Hoffmann is a Research Physicists at the Air Force Research Laboratory (AFRL) in Albuquerque, NM 87111 USA (email: Ryan.Hoffmann@Kirtland.af.mil ).

JR Dennison is a professor in the Department of Physics at Utah State University in Logan, UT 84322 USA (phone: 435-797-2936; fax: 435-7972492; e-mail: JR.Dennison@usu.edu). JR.Dennison@usu.edu). net charge accumulates in spacecraft components in response to incident electron, ion, and photon fluxes. The material resistivity, $\rho$, determines how quickly that charge is dissipated. Due to their high mobility, incident electrons from the space plasma play a more significant role in electron yield and in resulting spacecraft charging than do positively charged ions. For this reason the focus of this study is on electron interactions and ion and photon interactions are neglected.

The electron emission properties of electrically insulating materials, as a function of incident electron charge, are central to modeling spacecraft charging. Insulating materials generally exhibit higher yields than conducting materials, and accumulated charge cannot be easily dissipated. Therefore, insulating materials can become very efficient at collecting and storing charge. This becomes a very dynamic problem, as electron emission in insulators is complicated by the fact that the emission mechanisms themselves can be influenced by accumulated surface and bulk charge. In addition, the conductivity of the material can be modified by the energy deposited by the incident electron [1]. The net charge that a material will obtain is dictated by the complex interplay of all of these processes.

Because of the difficulty in measurement, yield is often neglected as a significant contributor to the effect of spacecraft charging. Instead, we in the spacecraft charging community, tend to focus on resistivity because of its relative ease of measurement and the ability of the resistivity parameter to be easily modified in models of specific applications.

\section{YIELD MEASUREMENT METHODS}

This work outlines three methods developed by the Utah State University (USU) Materials Physics Group (MPG) to measure the electron-induced electron yield of materials with resistivities ranging from conductors with $\rho \rightarrow 0$ to extreme insulators with $\rho \rightarrow \infty$ and with maximum total yields ranging from $\sigma_{\max }<1$ to $\sigma_{\max } \sim 40$. Thus, a combination of these methods span the full spectrum of spacecraft materials testing that needs to be performed for full inclusion of electron yields into modeling codes developed to predict spacecraft charging potentials.

\section{A. DC Yield Method}

A DC method with a continuous, low-current beam of electrons of energy $E_{b}$ is used to measure electron emission and electron yield from conductors, semiconductors, and modest insulators with $\rho<10^{17} \Omega$-cm.. Charge added to or 
removed from the sample, via electron emission, can be rapidly replaced by connecting the sample to ground [2, 3]. The fully encased hemispherical grid retarding field detector, shown in Fig. 1, facilitates high accuracy measurements of absolute yields; such measurements of absolute yields with very high accuracy (on the order of $\pm 2 \%$ ) are not possible using other common instrumentation and methods $[4,5]$. It also allows the application of bias to each of the discrete elements of the detector. These biases allow for the discrimination of secondary (SE) and backscatter electrons (BSE) and measurement of electron emission spectra, in contrast to the more typical method of only measuring the biased sample current. The individually biased elements of the HGRFA detector also allow for extensive instrument characterization and calibration. A thorough discussion of the DC system and methods is given by Thomson [5] and other references $[4,6]$.

Two electron sources provide electron energy ranges from $\sim 20 \mathrm{eV}$ to $\sim 30 \mathrm{keV}$ and incident electron currents (1 to $500 \mathrm{nA}$ or $<0.1 \mathrm{nA} / \mathrm{cm}^{2}$ to $50 \mu \mathrm{A} / \mathrm{cm}^{2}$ current densities) with pulsing capabilities ranging from 10 ns to continuous emission. The low-energy Staib electron gun operates at incident electron energies of $\sim 30 \mathrm{eV}$ to $5000 \mathrm{eV}$ with a maximum beam current of $\sim 100 \mathrm{nA}$ and a $<0.1 \mathrm{~mm}$ diameter minimum beam spot. The high-energy Kimball electron gun operated at incident electron energies of $3.5 \mathrm{keV}$ to $30 \mathrm{keV}$ with a typical beam current of $\sim 20 \mathrm{nA}$ and a $500 \mu \mathrm{m}$ minimum diameter beam spot.

For electron yield measurements on conductors, a continuous incident beam is directed on the sample and the currents on the detector (see (a) in Fig. 1), suppression grid ((b) in Fig.1), inner-grid ((c) in Fig. 1), sample ((d) in Fig. 1) and stage ((e) in Fig.1) are measured using custom electrometers [7]. A $50 \mathrm{~V}$ bias, relative to the suppression grid, is maintained at all times on the detector to insure that all electrons that are able to penetrate the grids are then collected. Grounding the grids through the electrometer facilitates the measurement of the total yield $\sigma$ by allowing all emitted electrons to be collected. A -50 V bias relative to the sample is then applied to the suppression grid allowing only the BSE with energies $>50 \mathrm{eV}$ to reach the detector, thus determining the backscatter electron (BSE) yield. The secondary yield is calculated as the difference between the BSE and the total yields.

A representative yield curve for of conductor yields for polycrystalline gold is shown in Fig.2 [4]. $E_{1}$ and $E_{2}$ are the first and second crossover energies. The yield peak, $\sigma_{\max }$, is the maximum yield and occurs between the crossover energies at $E_{\max }$.

\section{B. Effects of Charge Accumulation on Yields}

Figure 3 shows the pronounced effects of accumulated charge on yield measurements of insulating materials, even when low fluence pulsed methods are employed. Several distinct areas (Zones 1-6) are identified for the curves:

Zone 1: $E_{b}<E_{1}$. Yields are not affected appreciably by the positive charge.



Figure 1. (a) Simplified cross-section of Hemispherical Grid Retarding Field Analyzer (HGRFA) used for electron emission detection in all methods. (b) Schematic of HGRFA. (a) Solid hemispherical collector held at $+50 \mathrm{~V}$ relative to the bias grid to attract all electrons that penetrate the bias grid. (b) Bias grid used to discriminate electron energies coming from within. (c) Inner grid used to provide a uniform electric field and shield from unwanted edge effects. (d) Sample stage comprises the hemispherical and sample mounting platform. (e) The sample is held in the center of hemisphere.

Zone 2: $E_{1}>E_{b}>E_{2}$, where $\sigma>1 . \quad \sigma$ is depressed due to positive sample charging and the subsequent reattraction of some low energy SE's. BSE yield is unaffected by relatively small positive surface voltage, $V_{s}$. 
Zone 3: $E_{2}<E_{b}<\sim 1200 \mathrm{eV}$ is above the true uncharged $\sigma$ depicted by the green line. This may result from extra SE from the depletion region that are emitted due to the large electric field from the negative layer at penetration depth $R$.

Zone 4: $1200 \mathrm{eV}<E_{b}<1500 \mathrm{eV}$. Many of the SE's are accelerated up into the BSE range $(>50 \mathrm{eV})$ by large negative surface potentials, $\left|V_{s}\right|>50$.

Zone 5: $\sigma$ is enhanced because of the negative surface potential and the acceleration of the SE into BSE range.

Zone 6: $\sigma$ and BSE yields largely return to the idealized uncharged green curve, as essentially all SE have been accelerated and converted to BSE.

\section{Pulsed Yield Method}

The system employed at USU to measure electron emission from insulators (see Fig. 4) uses the same fully encased HGFRA detector and electron sources employed for DC measurements, in concert with methods to control the deposition and neutralization of charge [4, 8-10]. This is accomplished by minimizing the amount of charge used in the probe beam by using a pulsed, low-fluence beam rather than the continuous beam used for conductors. Typically, charge deposition is minimized by using a low-current beam focused on a sample area of $\sim 2.3 \pm 0.2 \mathrm{~mm}^{2}$ that is delivered in short pulses of $\sim 5 \mu \mathrm{sec}$. The pulsed system uses custom detection electronics with fast (1-2 $\mu$ s rise time) sensitive/low noise $\left(10^{7}\right.$ V/A / 100 pA noise level) ammeters [5]. Great pains have been taken to minimize overall system noise to reach the capabilities listed above. These efforts have included AC power filtering, increased cable shielding, identification and removal of problematic noise sources, identification and removal of ground loops. The system is capable of measuring an incident pulse of $6 \cdot 10^{3}$ electrons $/ \mathrm{mm}^{2}$. A simple approximation determined the charge density from such a pulse to be $6 \cdot 10^{6}$ electrons $/ \mathrm{mm}^{3}$, assuming an isotropic distribution of electrons in the material from the surface to the penetration depth of $\sim 1 \mu \mathrm{m}$. For perspective, this electron density can be compared to that of intrinsic silicon with a free carrier density of $\sim 6 \cdot 10^{9}$ electrons $/ \mathrm{mm}^{3}$.

Pulsing the beam minimizes the deposited charge, but insulators can store charge for a very long time. As a result, significant charge will accumulate on the material after several pulses. By using a pulsed, low-fluence electron probe pulse the deposited charge is minimized, but after repeated pulses charge will accumulate on the material and cause an unacceptable level of modification to the yield measurements. To counter this, a low energy $(3 \mathrm{eV})$ defocused flood gun is used to dust the surface with electrons and neutralize positive charge accumulation. The electron flood gun used for charge neutralization can also provide a focused low-energy ( $1 \mathrm{eV}$ to $200 \mathrm{eV}$ ) source. The data in Fig. 5 shows the modification of the total yield for successive pulses, with and without low energy electron flooding. Note that the unneutralized yield curve asymptotically approaches unity as the charged sample reattracts emitted electrons. The neutralized yield remains nearly constant, with a slight increase attributed to increased defects generated by the incident radiation flux. This clearly

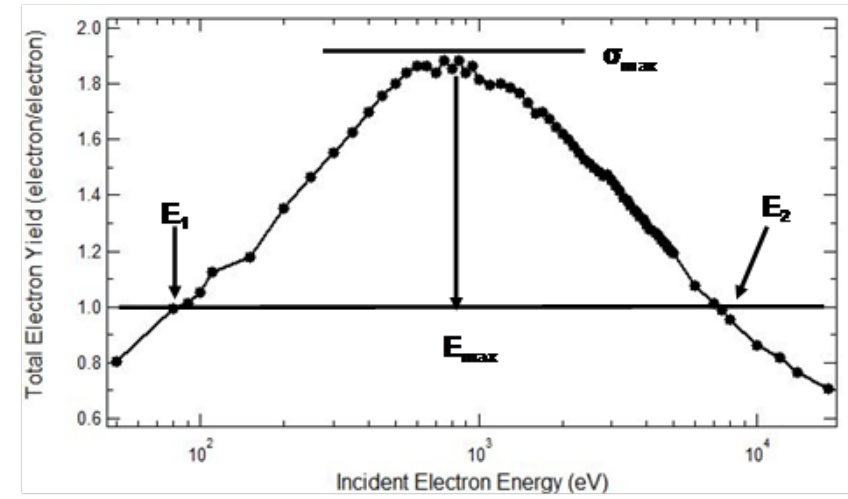

Figure 2. Total electron yield of polycrystalline Au as a function of incident energy. Data were taken using DC Yield Method.

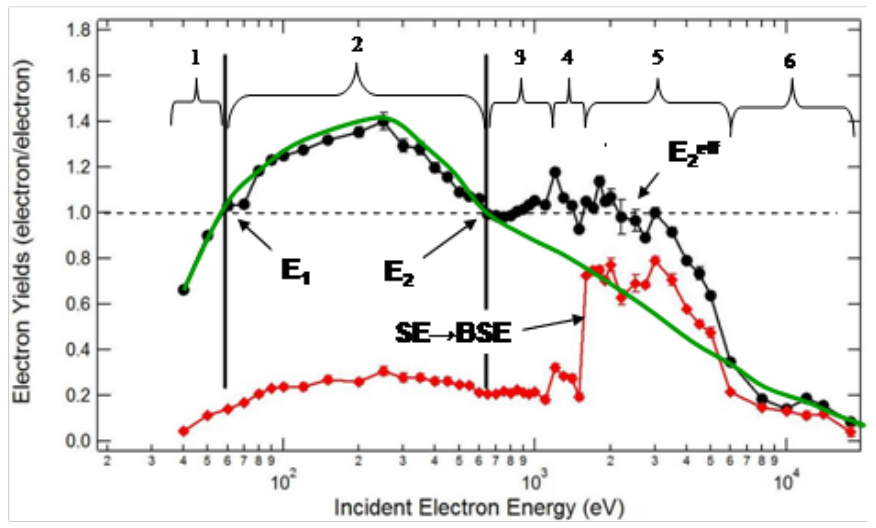

Figure 3. Total (black) and backscattered (red) electron yield for CP1 (a modified, more conductive form of polyimide) showing pronounced regions of charging behavior. The (green) curve is the estimated "intrinsic" or uncharged yield curve.

demonstrates the method of electron flood charge neutralization is effective.

Because surface charging is a function of incident flux density and not simply incident fluence, a careful characterization has been performed on the electron sources. By measuring the beam profile, and establishing controller settings for the full energy range, we have ensured that the spot size (and consequently flux density) is consistent at $1.7 \pm 0.3 \mathrm{~mm}$ FWHM over the entire yield curve. This is a departure from the work previously performed with this instrument, when spot size ranged from 0.3 to $1.5 \mathrm{~mm}$ in diameter [5, 11].

Minimizing the incident charge fluence $\left(\sim 5 \mathrm{fC} / \mathrm{mm}^{2}\right)$ with low flux $\left(\sim 1 \mathrm{nA} / \mathrm{mm}^{2}\right)$ and short duration pulses $(\sim 5 \mu \mathrm{s})$ and discharging the material after every pulse to prevent charge accumulation has proven to be effective when the material has both a high yield and high resistivity.. The data for Kapton HN in Fig.6 show total, SE, and BSE yield curves taken with the pulse yield system. This material has a high resistivity of $10^{19}$ $\Omega$-cm, with a corresponding charge decay time of $>10^{6} \mathrm{~s}$. Since this decay time is much longer than the few hrs required to measure a pulsed yield curve, the material is effectively a perfect charge integrator. There is no evidence of charge modified yield in Fig. 6 below $E_{b}>E_{2}$. Above this energy, negative surface potentials accelerate the SE's peaked at $\sim 5$ 




Figure 4. Block diagram of the pulse yield data acquisition and control system.

$\mathrm{eV}$ to energies $>50 \mathrm{eV}$ and these electrons begin to be measured as BSE's. This is to be expected, because our electron flood discharge methods do not dissipate negative charge accumulation.

The upper and lower bounds for resistivity of applicability for the Pulsed Yield Method are relatively easy to establish. The Pulsed Yield Method is applicable to materials with resistivities approaching zero for the lower bound. However, this method is not often used to test low resistivity materials, as it generally has greater error and is much more time intensive than the DC Yield Method. The Pulsed Yield Method can be effectively used for materials with resistivities approaching $\infty$. In practice, this upper limit is set by the isotropic extra-galactic cosmic background radiation flux, which is of high enough energy that it penetrates the atmosphere and so is not be appreciably attenuated by a vacuum test chamber or a spacecraft; it is essentially the same in all space environments or terrestrial laboratories. This cosmic radiation will excite some electrons into the conduction band and so will produce a constant lower bound to conductivity from radiation induced conductivity. A crude calculation-assuming a worldwide average natural background radiation dose for a human being from the cosmic ray background of $\sim 0.3 \mathrm{mSv}$ per year [12] and a typical biological radiation weighting factor of $1 \mathrm{~Gy} / \mathrm{Sv}$-predicts an annual dose of $\sim 46 \mathrm{mRad}$ and an average dose rate of $1 \cdot 10^{-9}$ $\mathrm{Rad} / \mathrm{s}$. For typical polymers at room temperature [13], this corresponds to a cosmic background RIC of $\sim 4 \cdot 10^{-23}(\Omega-\mathrm{cm})^{-1}$; at $100 \mathrm{~K}$ for typical polymers [13] this background RIC is $\sim 4 \cdot 10^{-27}(\Omega-\mathrm{cm})^{-1}$. Therefore, an upper limit on resistivity for any materials can be set at $\sim 10^{24} \Omega$-cm, with a charge decay time of hundreds of years.

The limits of the Pulsed Yield Method in terms of total yield are illustrated by the yield curve taken for polycrystalline aluminum oxide ceramic. As can be seen from the black data in Fig. 7. $\mathrm{Al}_{2} \mathrm{O}_{3}$ has a much higher yield than that of Kapton, and as $E_{b}$ approaches $\sim 200 \mathrm{eV}$, the yield increases until $\sigma>4$, above which $\sigma$ begins to fall off. At this point, the charge contained in each individual pulse is enough to cause a significant positive potential in the irradiated portion of the sample, and as a result SE's are reattracted to the surface and the total yield is reduced toward unity. As $E_{b}$ increases, the effect continues until the yield drops to $\sim 1$, at which point the probe pulse is no longer causing significant charging. This sets the upper limit of applicability for the Pulsed Yield Method as $\sigma_{\max }<4$; this could be increased if the incident pulse could be made smaller by further reducing the signal-to-noise ratio inherent in the system. As a lower bound for total yield this method is capable of measuring yields approaching zero.

\section{Composite Yield Method}

The Composite Yield Method or Yield Decay Method combines the low fluence Pulsed Yield Method and emission spectra data to determine the yield curves of high yield insulator that still charge under low current pulses. The Composite Yield Method overcomes this by measuring the response of the yield to incident charge and then using modeling to extrapolate to a minimally charged condition [14]. It is applicable to high yield insulators with typical yield $\sigma_{\max }$ 


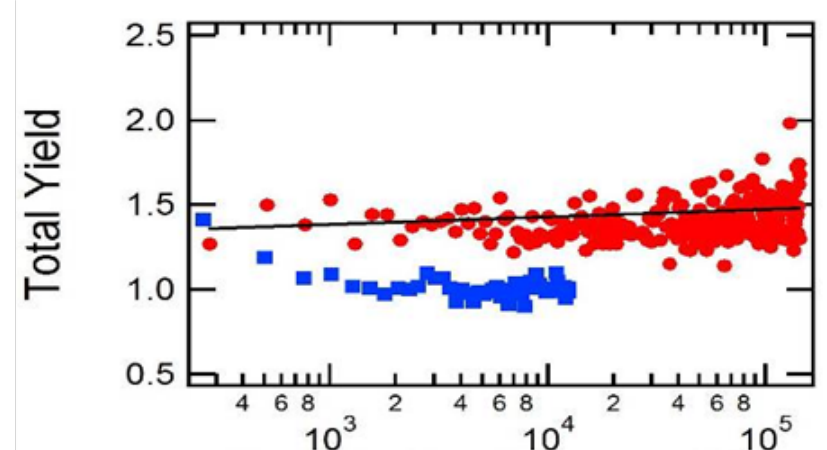

Total Incident Charge (fC)

Figure. 5 To show the effectiveness of low fluence pulsing coupled with low energy flooding two sets of data were taken on Kapton $\mathrm{HN}$ at $200 \mathrm{eV}$ incident energy, one with charge neutralizing flooding (red) and one without flooding (blue).

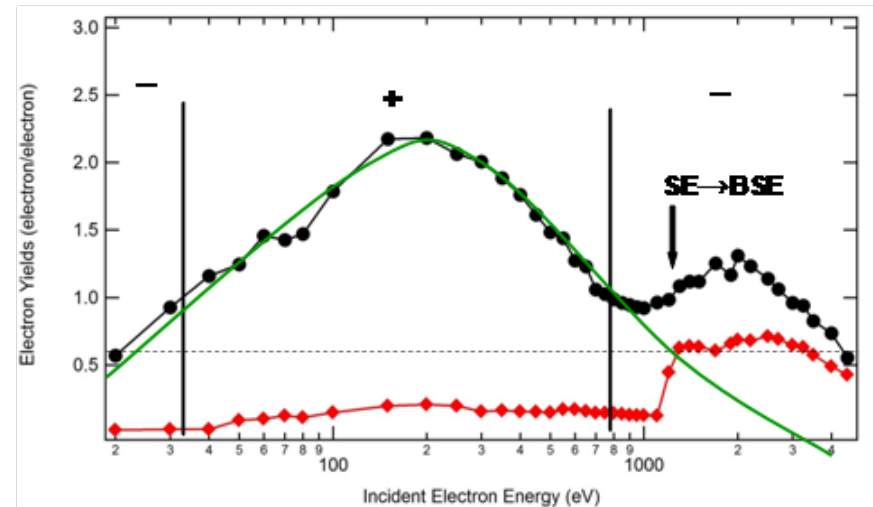

Figure 6. Total (black) and backscattered (BSE) (red) electron yield curves for Kapton HN taken with the pulsed yield system employing both low-fluence pulses and low-energy flooding. There is no evidence of charging up to $\sim 1100 \mathrm{eV}$, where the BSE yield abruptly increases. This is an indication that SE's are being accelerated to $>50 \mathrm{eV}$ due to negative potential within the material.

$>4$ and $\rho \rightarrow \infty(\Omega$-cm). The treatment given this method here is an overview of the concept, for a full explanation see Hoffmann [11, 14].

When a material is exposed to high-energy electron irradiation, electrons emitted from the material have a range of emission energies from $0 \mathrm{eV}$ up to the incident electron energy, $E_{o}$. Emitted electrons with energies $<50 \mathrm{eV}$ are assumed to be secondary electrons (SE's) originating from within the material. The escape energies of SE's depend on their production depth, as well as the energy-loss mechanisms and potential barriers experienced before exiting the material, but $50 \mathrm{eV}$ serves as a convenient demarcation as long as the incident electrons $E_{b}>50 \mathrm{eV}$. Backscatter electrons (BSE's) are electrons that elastically scatter from the material and have energies up to those of the incident electrons. Again, a convenient demarcation for BSE is that they have energies $>50 \mathrm{eV}$; this is valid as long as the incident beam $E_{b}>50 \mathrm{eV}$.

Measured emission spectra for Au are shown in Fig 8, along with a fit based on the Chung-Everhart model [15]. Between the total-yield crossover energies, $E_{1}$ and $E_{2}$, the magnitude of insulator charging is positive (since the total yield is greater

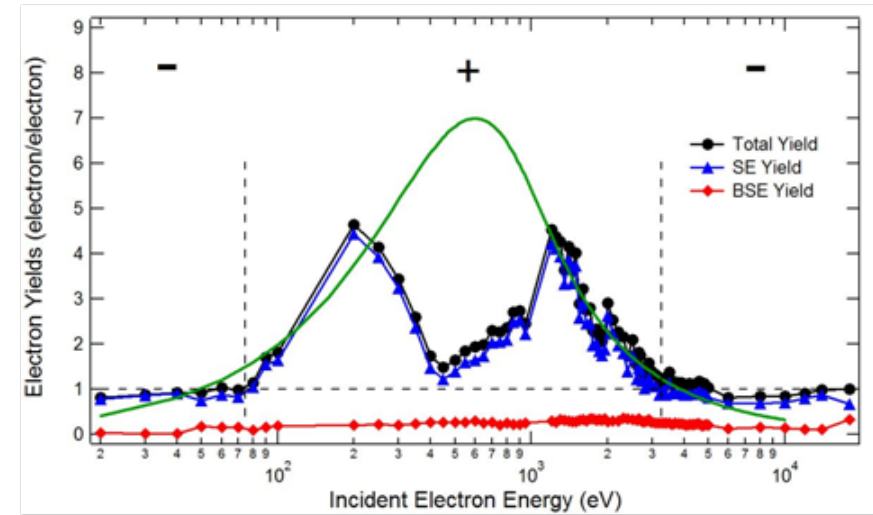

Figure 7. Yield of polycrystalline $\mathrm{Al}_{2} \mathrm{O}_{3}$ taken using the Pulsed Yield Method and predicted with the Composite Yield Method. The dual peak behavior between the crossover energies is evidence of positive charging as a significant fraction of the SE's are reattracted to the surface. The green line is a "best guess" for the "intrinsic" yield.

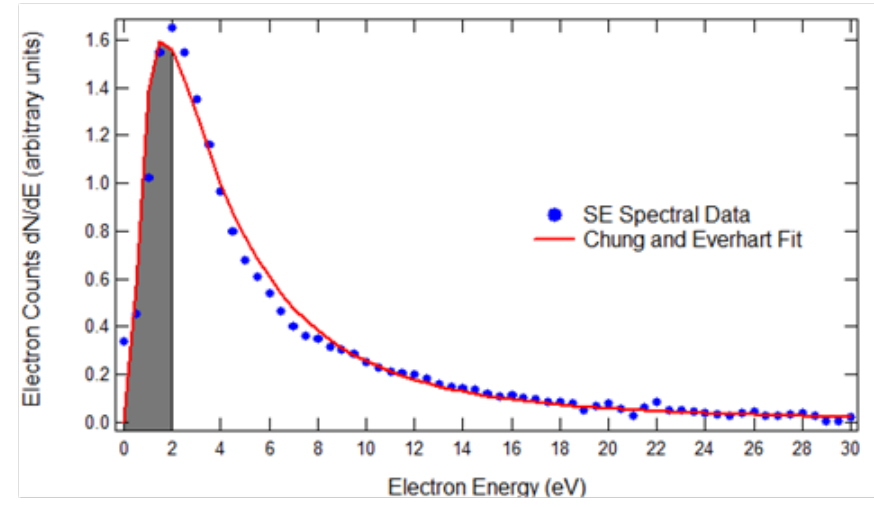

Figure 8. Secondary Electron (SE) emission spectra of polycrystalline $\mathrm{Al}_{2} \mathrm{O}_{3}$ fit with a model developed by Chung and Everheart (1975). The gray area represents the fraction of SE that would be reattracted to the surface with a $+2 \mathrm{~V}$ surface potential.

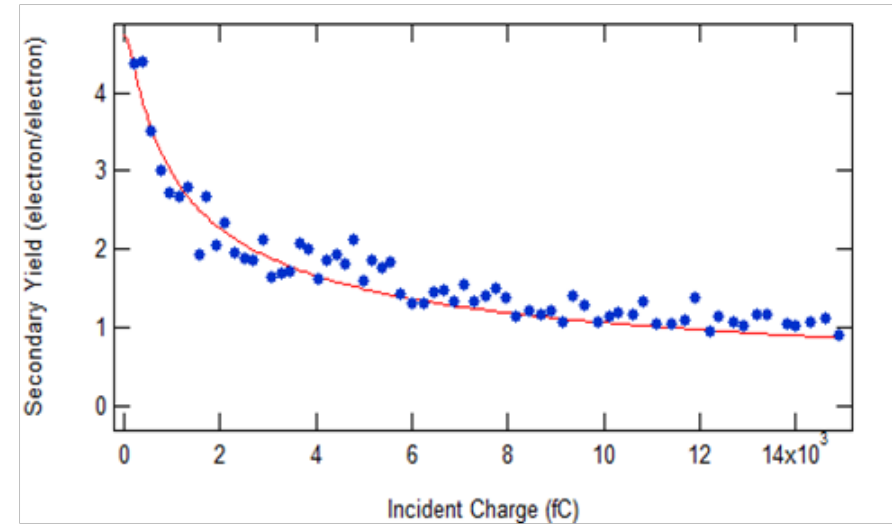

Figure 9. By measuring the yield as a material charges, the integrated Chung and Everheart model can be used to fit the yield as a function of incident charge. This allows an extrapolation of the yield to negligible incident charge.

than one), and due to the reattraction of low energy electrons, the insulator attains a steady-state surface potential of just a few volts positive. This positive charging increases the insulator surface potential barrier by an amount $e V_{s}$, where $V_{s}$ is the positive surface potential. Hence, the resulting SE yield emitted from a positively charged specimen can be expressed 
as an integral of the uncharged spectrum (taken at the same incident energy) with the integration limits extending from the positive surface potential up to the arbitrary $50 \mathrm{eV}$ limit of SE energy $[16,17]$.

An analytical solution to this integral gives an expression that describes the reattraction of SE's to the surface of a positively charged sample. The positive surface charging inhibits the escape of lower-energy SE's, thus suppressing the lower-energy portion of the SE spectrum (represented by the shaded area in Fig. 8). Consequently, only the unshaded area of the electron energy spectrum (above $e V_{s}$ ) contributes to the charged electron yield.

This yield decay model describes the modification of the total yield as a response to surface potential. While efforts are underway to measure the surface potential directly (Hodges 2010), there currently exists the capability to only measure the incident and return flux. To relate the surface voltage to the incident flux, the Dual Dynamic Layer Model (DDLM) [1822] has been employed to establish the relationship between the surface voltage and incident electron flux. This allows us to model the evolution the total yield as a function of the measured incident flux or as a function of the surface potential (what is calculated using the DDLM). These data and fit are shown in Fig. 10.

Using the Composite Yield Method, the total yield is measured as incident flux is increased and then these data are fit with a physics-based empirical model as the yield decays to unity This fit then allows the extrapolation of the yield to incident fluxes approaching negligible charge, the so called "intrinsic" yield. This extrapolation is only valid for the total yield at the incident energy of the decay curve.. In order to get the complete total yield curve, data must be taken for a spectrum of incident energies, fit with the yield decay model, and then extrapolated to a $0 \mathrm{~V}$ surface potential. These data are shown in green Fig. 10. The discrepancies between the measured data and the green "intrinsic" yield curve show where the pulsed yield method failed due to charging of the polycrystalline aluminum oxide.

This method is very time consuming and difficult to implement, but it has proven capability to measure materials with both high yield $>4$ and high resistivity $>10^{16} \Omega$-cm. This ability is due to the fact that this method does not attempt to minimize and dissipate charging as in the pulse yield method; rather it takes advantage of charging and models the evolution of the total yield, thus allowing predictions of an uncharged yield. To apply this method requires that the material must have a charge decay time constant of $>4 \mathrm{~s}$ (the experimental time frame to acquire one yield measurement at a given incident energy)—-with a corresponding resistivity $>10^{12} \Omega$ $\mathrm{cm}$ - such that charge is allowed to accumulate on the surface between measurements. Since there this method relies on a model of positive surface potential reattraction of SE's it cannot measure a total yield of 1 . The upper bound for total yield is set by the minimum flux contained in each probe pulse, or in other words how quickly the material charges and consequently how many point there are on the yield decay curve before it reaches unity. Based on this, we project that the

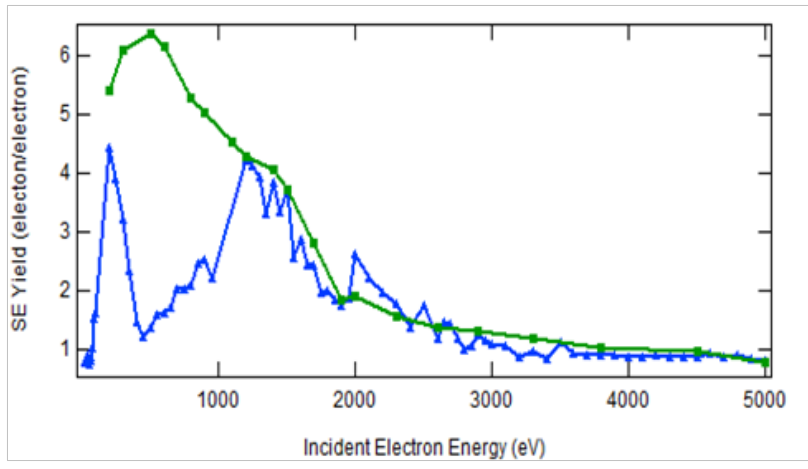

Figure 10. By producing many curves like the one in Fig. 10 and extrapolating them to zero incident charge, we obtain the "intrinsic" yield curve for high yield insulators shown in green (Hoffmann 2008).

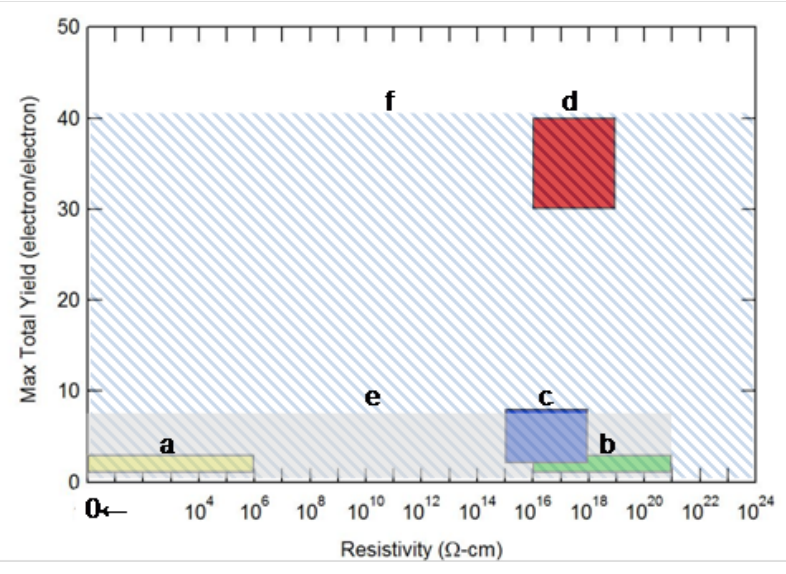

Figure 11. Description of measurable ranges of materials.

a) Low resistivity conductors and semiconductors.

b) Low yield insulators, such as polymers (e.g., Kapton or Mylar).

c) High yield insulators, such as metal oxide ceramics (e.g., $\mathrm{Al}_{2} \mathrm{O}$ or $\left.\mathrm{MgO}\right)$.

d) Extremely high yield negative electron affinity materials (e.g., diamond).

e) Range of material testing to date by USU Materials Physics Group.

f) Potential range of applicability of currently developed test methods.

Composite Yield Method can be applied to materials with $\sigma_{\max } \leq 40$.

\section{SUMMARY}

The parameters of maximum total yield and conductivity dictate the charging susceptibility. Figure 11 depicts the demonstrated and predicted capabilities of the three methods described here. These methods have demonstrated broad applicability to a wide variety of materials. Further, we predicted that these methods can be used to measure materials with any degree of charging susceptibility from conductors (low-yield; low-resistivity) to diamond (high-yield; highresistivity). The methods described herein are applicable over the entire range of both yield (from 0 to 40 ) and resistivity (from $0(\Omega-\mathrm{cm})$ to $>10^{24}(\Omega$-cm)) [11]. 
In the spacecraft charging community much attention is paid to a materials resistivity or in other words how the material stores charge. When a charging problem is encountered the first question asked is what is the resistivity and can it be changed. This myopic view neglects electron yield as the other primary mechanism for spacecraft charging. A primary reason for this one-sided approach is the fact that consistent, reliable, and repeatable yield data are not available in the literature, especially for insulators. The methods to acquire these data simply did not exist in the past, so researches were required to use poor quality yield data as inputs in charging codes. The methods and capabilities described herein have completely overcome this deficiency. We have demonstrated the ability to measure uncharged yields from polymers such as Kapton $\mathrm{HN}$ as well as ceramics such as polycrystalline aluminum oxide. Further we predict that it will be possible to use these methods on even the most challenging high yield materials, the so called negative electron affinity materials such as diamond and certain doped cover glass materials.

\section{REFERENCES}

[1] J. Dennison, R. Hoffmann, J. Roth, and A. Sim, "Effects of Radiation Induced Conductivity on Samples Charged by Particle Beams," in 2008 Joint Fall Meeting of the Texas and Four Corners Sections of APS, AAPT, and Zones 13 and 16 of SPS, and the Societies of Hispanic and Black Physicists, 2008.

[2] E. Baroody, "A theory of secondary electron emission from metals," Physical Review, vol. 78, pp. 780-787, 1950.

[3] H. Bruining and J. De Boer, "Secondary Electron Emission: Part I. Secondary Electron Emission of Metals," Physica, vol. 5, pp. 17-30, 1938.

[4] N. Nickles, W.-Y. Chang, and J. R. Dennison, "Hemispherical grid retarding field analyzer for absolute measurement of secondary and back-scattered electron yields," in American Physical Society March Meeting 2001 Seattle, Washington: Bull. Am. Phys. Soc. 46(1) Part I, 443, (2001), 2001.

[5] C. Thomson, "Measurements of the Secondary Electron Emission Properties of Insulators," in Physics. vol. Ph.D. Logan, UT: Utah State University, 2004.

[6] W. Chang, J. Dennison, P. Judd, and U. Logan, "Measurements of Electronic Properties of Conducting Spacecraft Materials with Application to the Modeling of Spacecraft Charging," in 38th American Institute of Aeronautics and Astronomics on Aerospace Sciences, Reno, NV, 2000.

[7] J. R. Dennison, A. Chang, N. Nickles, C. D. Thomson, J. Kite, and R. Davies, "Ground-based Measurements Simulating Space Environment Interaction of Materials and SEE Spacecraft Charging Materials Database for Spacecraft Charging Modeling," in International Union of Radio Scientists (URSI) National Radio Science Meeting University of Colorado, Boulder, Colorado, 2002.

[8] J. Dennison, C. Thomson, J. Kite, V. Zavyalov, and J. Corbridge, "Materials characterization at Utah State University: facilities and knowledgebase of electronic properties of materials applicable to spacecraft charging," in Proceedings of the 8 th Spacecraft Charging Technology Conference, NASA Marshall Space Flight Center, Huntsville, Al, 2003, p. 15.

[9] W. Y. Chang, N. Nickles, J. R. Dennison, and C. D. Thomson, "An Improved Database of Electronic Properties of Spacecraft Materials for Modeling of Spacecraft Charging," in 7th
Spacecraft Charging Technology Conference Noordwijk, The Netherlands, 2000.

[10] C. D. Thomson, V. Zavyalov, and J. R. Dennison, "Instrumentation for Studies of Electron Emission and Charging from Insulators," in Proceedings of the 8th Spacecraft Charging Technology Conference, NASA Marshall Space Flight Center, Huntsville, Al, 2003, p. 15.

[11] R. Hoffmann, "Electron-Induced Electron Yields of Uncharged Insulating Materials " in Physics. vol. M.S. Logan, UT Utah State University 2010, p. 183.

[12] M. Thorne, "Background radiation: natural and man-made," Journal of Radiological Protection, vol. 23, p. 29, 2003.

[13] J. Dennison, J. Gillespie, J. Hodges, R. Hoffmann, J. Abbott, A. Hunt, and R. Spalding, "Radiation Induced Conductivity of highly-Insulating Spacecraft Materials," in American Institute of Physics Conference Proceedings Series, Melveille, NY, 2009, 2009, pp. 203-208.

[14] R. Hoffmann, J. Dennison, C. Thomson, and J. Albretsen, "Low-Fluence Electron Yields of Highly Insulating Materials," IEEE Transactions on Plasma Science, vol. 36, pp. 2238-2245, 2008.

[15] M. Chung and T. Everhart, "Simple calculation of energy distribution of low energy secondary electrons emitted from metals under electron bombardment," Journal of Applied Physics, vol. 45, p. 707, 1974.

[16] N. Nickles, R. Davies, and J. Dennison, "Applications of Secondary Electron Energy-and Angular-Distributions to Spacecraft Charging," in Proceedings of the 6th Spacecraft Charging Conference, AFRL Science Center, Hanscom AFB, MA, USA.,, 1998, pp. 275-280.

[17] L. Reimer, "Scanning electron microscopy: physics of image formation and microanalysis," Measurement Science and Technology, vol. 11, p. 1826, 2000.

[18] Cazaux, J., 1999, "Some Considerations on the Secondary Electron Emission, $\Delta$, from E Irradiated Insulators," J. Appl. Phys. 85, 1137.

[19] Cazaux, J., 2003, "Scenario for Time Evolution of Insulator Charging under Various Focused Electron Irradiations," J. Appl. Phys. 95, 731.

[20] Melchinger, A., and S. Hofmann, 1995, "Dynamic Double Layer Model: Description of Time Dependent Charging Phenomena in Insulators under Electron Beam Irradiation," J. Appl. Phys. 78, 6224.

[21] Meyza, X., D. Goeuriot, C. Guerret-Piecourt, D. Treheux, and H. Fitting, 2003, "Secondary Electron Emission and SelfConsistent Charge Transport and Storage in Bulk Insulators: Application to Alumina," J. Appl. Phys. 94, 5384. 